\title{
Manual Pheresis
}

National Cancer Institute

\section{Source}

National Cancer Institute. Manual Pheresis. NCI Thesaurus. Code C133334.

Procedures such as plasmapheresis, plateletpheresis, and leukapheresis, in which unneeded portions of the whole blood are returned to the donor. 